\title{
Intervenções Percutâneas no Pós-Operatório Tardio de Cirurgia de Correção Atrial para Transposição das Grandes Artérias
}

\author{
Santiago Raul Arrieta', Juliana R. Neves ${ }^{1}$, Gustavo Andrade' ${ }^{1}$, Catarina Cavalcanti², Mônica Fiori² \\ Renata de Sá Cassar', Cleusa Lapa'
}

\section{RESUMO}

A obstrução dos túneis intra-atriais, no pós-operatório tardio de cirurgia de correção atrial para o tratamento da transposição das grandes artérias, não é uma complicação infreqüente, podendo até, em alguns casos, ser fatal. O tratamento cirúrgico de tais lesões pode apresentar altos índices de morbidade e mortalidade. Recentemente, o tratamento percutâneo mediante implante de stent ou por meio de angioplastia com cateter-balão tem sido utilizado com bons resultados. Relatamos dois casos de tratamento percutâneo de obstruções dos túneis intra-atriais sistêmico e pulmonar.

DESCRITORES: Contenedores. Transposição dos grandes vasos. Átrios do coração, cirurgia.
$\mathbf{N}$ as décadas de 1970 e 1980, a cirurgia para correção da transposição das grandes artérias (TGA) era chamada de "correção atrial ou fisiológica" (cirurgias de Senning ou Mustard), que consistia na criação de túneis intra-atriais para levar o sangue das veias cavas para a valva mitral e das veias pulmonares para a valva tricúspide. Apesar de ambas as técnicas apresentarem baixo índice de mortalidade, algumas complicações imediatas e tardias podem ser observadas ${ }^{1,2}$. Após a introdução da correção anatômica (cirurgia de Jatene $)^{3}$ para a TGA, a correção fisiológica, no plano atrial, foi praticamente abandonada, sendo utilizada na atualidade apenas em casos selecionados ${ }^{4}$.

As complicações tardias mais freqüentes das cirurgias de Senning ou Mustard incluem obstruções dos túneis intra-atriais (sistêmicos ou pulmonares), disfunção

\footnotetext{
1 Instituto Materno Infantil Prof. Fernando Figueira - Recife, PE. 2 Hospital Oswaldo Cruz - Recife, PE.

Correspondência: Santiago Raul Arrieta. Rua Amazonas, 70 - apto. 701 - Boa Viagem - Recife, PE - CEP 51011-020

E-mail: raularrieta@imip.org.br

Recebido em: 8/10/2007 - Aceito em: 8/11/2007
}

\section{SUMMARY}

Cardiac Interventions in the Late Follow-Up of Atrial Repair for Transposition of the Great Arteries

Obstruction of the venous pathways after atrial repair for transposition of the great arteries is not uncommon and can be, in some cases, fatal. The surgical treatment of these complications is a high-risk procedure. Nowadays, percutaneous balloon angioplasty or stenting have been used with good results. We report two cases of percutaneous treatment of systemic and pulmonary "baffle" obstructions.

DESCRIPTORS: Stents. Transposition of great vessels. Heart atria, surgery.

ventricular direita, insuficiência das valvas atrioventriculares, obstrução da via de saída do ventrículo esquerdo, arritmias e até, em alguns casos, morte súbita ${ }^{5,6}$.

A estenose dos túneis intra-atriais é uma complicação não infreqüente ${ }^{7}$, sendo a principal causa de reoperação, porém com altos índices de morbidade e mortalidade $^{5,6}$.

Neste artigo, relatamos o tratamento percutâneo de dois pacientes que apresentaram quadro clínico grave decorrente de estenose dos túneis intra-atriais.

\section{CASO 1}

Trata-se de paciente do sexo masculino, de 21 anos de idade, portador de TGA simples, que foi submetido a correção atrial (Mustard) no primeiro ano de vida. Durante seu acompanhamento ambulatorial nos primeiros oito anos, não apresentou problemas significativos e a família decidiu abandonar o seguimento. Em sua volta ao hospital de origem, após 14 anos, o paciente referia dispnéia progressiva (classe funcional III-IV) e cianose acentuada. O exame físico na admissão evidenciou edema em membros inferiores, estase 
Arrieta SR, et al. Intervenções Percutâneas no Pós-Operatório Tardio de Cirurgia de Correção Atrial para Transposição das Grandes Artérias. Rev Bras Cardiol Invas. 2008;16(2):234-238.

jugular 4/4, hepatomegalia grave e ascite significativa; o ritmo cardíaco era regular, com bulhas normofonéticas, sem sopros. Na ausculta respiratória, apresentava estertores subcrepitantes esparsos com taquipnéia leve. A saturação arterial era de $60 \%$ e a pressão arterial, de $110 / 70 \mathrm{mmHg}$. A radiografia de tórax demonstrou área cardíaca discretamente aumentada e campos pulmonares limpos. Iniciou-se tratamento para insuficiência cardíaca congestiva e realizou-se ecocardiograma transtorácico, que evidenciou sinais de estenose no túnel da veia cava superior (VCS) e ausência de fluxo através da veia cava inferior $(\mathrm{VCl})$. A tunelização venosa pulmonar encontrava-se aparentemente sem sinais de obstrução. Havia boa função biventricular, com valvas atrioventriculares competentes. Após 48 horas de medicação endovenosa, o paciente foi transferido para nossa instituição para a realização de cateterismo cardíaco, com o objetivo de confirmação diagnóstica e avaliação terapêutica.

Realizou-se cateterismo cardíaco sob anestesia geral, com intubação orotraqueal e punção das veias femorais direita e esquerda e jugular direita. Na injeção de contraste observou-se que ambas as veias femorais estavam ocluídas, tendo sido evidenciada circulação colateral significativa com drenagem em plexo paravertebral, sem comunicação com a $\mathrm{VCl}$. $\mathrm{Na}$ angiografia da VCS, notou-se estenose acentuada do túnel, medindo aproximadamente $5 \mathrm{~mm}$ de diâmetro, com circulação retrógrada através da veia ázigo, drenando num sistema paravertebral e dando origem a conexões venosas para o átrio direito (arterial), o que justificava a cianose do paciente (Figuras $1 \mathrm{~A}$ e 1B). Optou-se pela realização de angioplastia e implante de stent através da veia jugular direita no túnel da VCS. Após passagem, através da lesão, de uma guia extra-stiff $260 \mathrm{~cm}$ (Medi-Tech, Boston Scientific, Massachusetts, Estados Unidos), foi introduzido um stent Max LD 36 mm (Ev3, Minnesota, Estados Unidos) montado num balão MaxiLD 18/40 (Johnson \& Johnson-Cordis Corp., Florida, Estados Unidos) através de uma bainha $11 \mathrm{Fr}$ tipo Mullins Check-Flo (Cook Inc., Indiana, Estados Uniods). Após liberação do stent, o paciente apresentou significativa melhora hemodinâmica (Tabela 1) e angiográfica (Figura 1C), com inversão do fluxo pela veia ázigo e aumento da saturação para 96\%. Em decorrência da recuperação rápida do paciente e da impossibilidade de acesso ao túnel da $\mathrm{VCl}$, optou-se pela abordagem do mesmo num segundo tempo.

Na evolução imediata, o paciente se recuperou rapidamente, com perda de $9 \mathrm{~kg}$ de peso nas primeiras 36 horas, e recebeu alta hospitalar quatro dias após o procedimento em uso de ácido acetilsalicílico $325 \mathrm{mg} /$ dia e diuréticos.

Após um ano do procedimento inicial, o paciente permanecia em classe funcional I; porém, durante o acompanhamento, apresentou hemorragia digestiva alta,

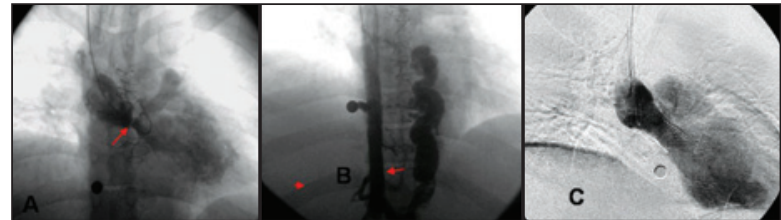

Figura 1 - Implante de stent no túnel da veia cava superior. A Angiografia do túnel da veia cava superior, evidenciando significativa estenose do túnel (seta), com dilatação pré-estenótica moderada. B: Circulação colateral da veia ázigo (seta) para plexo paravertebral e deste para o átrio direito. C: Após implante de stent, com nítida melhora do sítio da estenose.

TABELA 1

Dados hemodinâmicos do caso 1 , demonstrando as variáveis hemodinâmicas antes e após implante de stent no túnel da veia cava superior

\begin{tabular}{lcc} 
& Pré-intervenção & Pós-intervenção \\
\hline$\varnothing$ túnel VCS & $5 \mathrm{~mm}$ & $18 \mathrm{~mm}$ \\
$\mathrm{P} \vee C S$ & $28 \mathrm{mmHg}$ & $13 \mathrm{mmHg}$ \\
Sat $\mathrm{O}_{2}$ & $65 \%$ & $96 \%$ \\
\hline
\end{tabular}

$\varnothing$ túnel VCS = diâmetro do túnel da veia cava superior; P VCS = pressão na veia cava superior; Sat $\mathrm{O}_{2}=$ saturação arterial de oxigênio.

sendo evidenciada úlcera gástrica e presença de varizes esofágicas moderadas à endoscopia digestiva. Ao exame físico, notou-se presença de ascite discreta e quadro grave de varizes em membros inferiores. Ao ecocardiograma transtorácico, o stent no túnel da VCS estava bem posicionado e sem gradiente residual, e o fluxo pelo túnel da $\mathrm{VCl}$ era quase ausente. O paciente foi novamente encaminhado para o Laboratório de Hemodinâmica, para avaliações diagnóstica e terapêutica. Durante o novo cateterismo, foi realizada punção jugular direita e, em decorrência da obstrução das veias femorais, decidiu-se pela realização de punção trans-hepática com agulha Chiba $20 \mathrm{~cm}$ (Cook Inc., Indiana, Estados Unidos). Na angiografia da VCS, o stent encontrava-se com fluxo livre e sem sinais de deslocamento, e a veia ázigo apresentava fluxo normal proveniente dos membros inferiores. A angiografia da VCI por via trans-hepática demonstrou oclusão desse vaso, antes da origem das veias renais, com oclusão completa da veia renal direita, não sendo visualizado à fluoroscopia o sistema pielocalicial direito (Figuras $2 \mathrm{~A}$ e $2 \mathrm{~B}$ ).

No átrio, foi observada estenose crítica $(4 \mathrm{~mm})$ do túnel (Figura 2C), com abaulamento considerável do retalho utilizado para tunelização e retenção de contraste, evidenciando lentidão do fluxo sanguíneo e aumento significante da pressão venosa (Tabela 2). Em decorrência dos achados hemodinâmicos e diante da possibilidade de trombose total da $\mathrm{VCl}$ ou da outra veia 


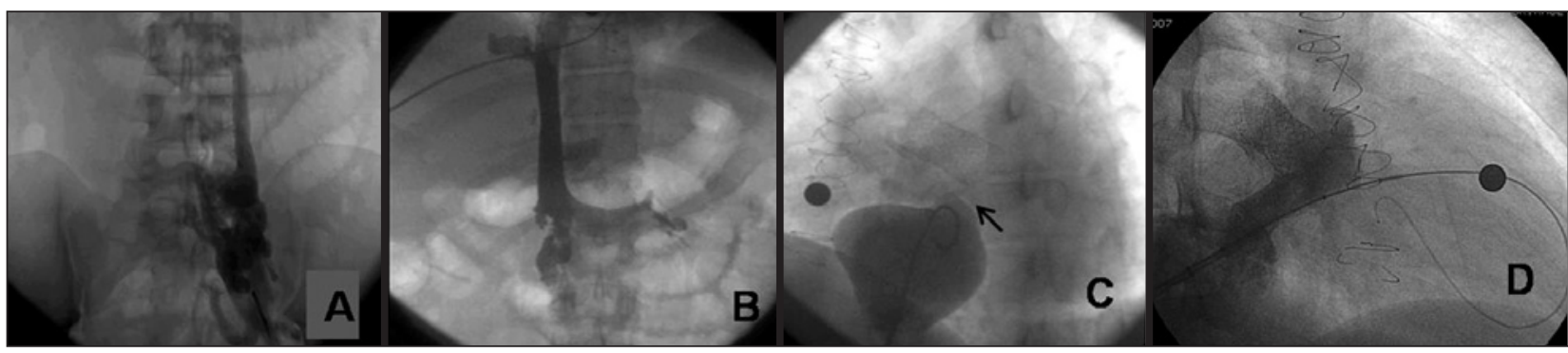

Figura 2 - Implante de stent no túnel da veia cava inferior. A: Oclusão da veia femoral esquerda com drenagem através de plexo paravertebral. B: Angiografia trans-hepática da veia cava inferior, demonstrando oclusão total da mesma antes da origem das veias renais e oclusão total da veia renal direita. C: Angiografia do túnel da veia cava inferior, evidenciando estenose grave (seta) na chegada ao átrio esquerdo, com dilatação acentuada do túnel antes do local da estenose. D: Após implante de stent, com nítida melhora do local da estenose.

TABELA 2

Dados hemodinâmicos do caso 2 , demonstrando as variáveis hemodinâmicas antes e após implante de stent no túnel da veia cava inferior

\begin{tabular}{lcc}
\hline & Pré-intervenção & Pós-intervenção \\
\hline$\varnothing$ túnel $\mathrm{VCl}$ & $4 \mathrm{~mm}$ & $16 \mathrm{~mm}$ \\
$\mathrm{P} \mathrm{VCl}$ & $22 \mathrm{mmHg}$ & $12 \mathrm{mmHg}$ \\
$\mathrm{Sat} \mathrm{O}_{2}$ & $96 \%$ & $96 \%$ \\
\hline$\varnothing$ túnel $\mathrm{VCl}=$ & diâmetro do túnel da veia cava inferior; \\
$\mathrm{P} \mathrm{VCl}=$ pressão na veia cava inferior; Sat $\mathrm{O}_{2}=$ saturação \\
arterial de oxigênio.
\end{tabular}

renal, decidiu-se realizar angioplastia com implante de stent no túnel da $\mathrm{VCl}$. Através do acesso trans-hepático, utilizando uma bainha $10 \mathrm{Fr}$ tipo Mullins, CheckFlo, foi introduzido e posicionado no ventrículo esquerdo um guia extra-stiff 0,035" de 260 cm (MediTech, Boston Scientific, Massachusetts, Estados Unidos) e realizado implante de stent Genesis XD 2910 (Johnson \& Johnson-Cordis Corp., Florida, Estados Unidos) sobre balão MaxiLD 16/40 (Johnson \& JohnsonCordis Corp., Florida, Estados Unidos) (Figura 2D). Houve nítida melhora dos parâmetros hemodinâmicos; depois do procedimento, a bainha foi retirada e o trajeto hepático foi ocluído com duas molas Gianturco 35/5/5 (Cook Inc., Indiana, Estados Unidos). O paciente, posteriormente, foi transferido da UTI em uso de enoxaparina e recebeu alta hospitalar após 36 horas.

\section{CASO 2}

Trata-se de criança do sexo feminino, de 12 anos de idade e $35 \mathrm{~kg}$ de peso, portadora de TGA simples, que foi submetida a cirurgia de Senning aos seis meses de idade. Foi admitida no hospital de origem em decorrência de cansaço progressivo nos últimos dois anos, com marcada piora nos últimos seis meses (classe funcional III). Ao exame físico, apresentou taquidispnéia moderada, ritmo cardíaco regular, bulhas normofonéticas e ausência de sopros. Na ausculta respi- ratória, evidenciava estertores crepitantes bilaterais, com roncos esparsos. A radiografia de tórax mostrou área cardíaca normal, com significativa congestão pulmonar. O ecocardiograma transtorácico demonstrou fluxo normal na tunelização das veias cavas para o átrio esquerdo e sinais de estenoses na tunelização das veias pulmonares para o átrio direito. A função biventricular era normal e as valvas cardíacas não apresentavam anormalidades. Foi encaminhada então para nossa instituição para confirmação diagnóstica e avaliação terapêutica por meio do cateterismo cardíaco.

O cateterismo cardíaco foi realizado sob anestesia geral, com intubação orotraqueal, sendo puncionadas artéria e veia femorais direitas. Foi realizado cateterismo direito e esquerdo, e a medida das pressões evidenciou hipertensão pulmonar moderada, com hipertensão venocapilar grave (Tabela 3). As angiografias nas VCS e $\mathrm{VCl}$ demonstraram tunelização ampla, sem sinais de estenoses ou extravasamentos. Para a visualização do túnel das veias pulmonares foram realizadas angiografias nas artérias pulmonares, porém sem que fosse obtida imagem de qualidade adequada. Foi então realizada a cateterização do átrio venoso pulmonar de forma retrógrada, através da aorta, do ventrículo direito, da valva tricúspide e do átrio venoso pulmonar, obtendose angiografia em perfil esquerdo que evidenciou estenose significativa $(5 \mathrm{~mm})$ na tunelização das veias pulmonares (Figura 3A). Em decorrência da gravidade do quadro clínico e da natureza focal da lesão, decidiu-se realizar angioplastia com cateter-balão. Através da artéria femoral (7 Fr) e com um guia extra-stiff ST1 de 260 cm (Medi-Tech, Boston Scientific, Massachusetts, Estados Unidos) posicionado na veia pulmonar inferior esquerda, foi realizada pré-dilatação da lesão com cateter-balão Powerflex 12/40. Nova medição das pressões foi realizada com cateter MultiTrack 5 Fr (NuMed Inc., New York, Estados Unidos), evidenciando melhora parcial (queda do gradiente pressórico < 50\%). Posteriormente foi introduzido balão MaxiLD 18/40 (Johnson \& Johnson-Cordis Corp., Florida, Estados Unidos), sendo realizada nova angioplastia com alta pressão (12 atm) durante 10 segundos, em duas oportunidades, com melho- 
Arrieta SR, et al. Intervenções Percutâneas no Pós-Operatório Tardio de Cirurgia de Correção Atrial para Transposição das Grandes Artérias. Rev Bras Cardiol Invas. 2008;16(2):234-238.

TABELA 3

Dados hemodinâmicos do caso 2 , antes e após intervenção

\begin{tabular}{lcc}
\hline & Pré-intervenção, $\mathbf{~ m m H g}$ (média) & Pós-intervenção, mmHg \\
\hline P VCS & 13 & 13 \\
P VCI & 10 & 12 \\
VE & $65 / 12$ & - \\
AP & $55 / 30(39)$ & - \\
Cap & 30 & 16 \\
AVP & 15 & 16 \\
$\varnothing$ túnel VP $(\mathrm{mm})$ & 5 & 18 \\
VD & $80 / 15$ & $80 / 15$ \\
Ao & $80 / 40(54)$ & $110 / 60$ \\
\hline P VCS = pressão na veia cava superior; P VCI = pressão na veia cava inferior; VE = ventrículo esquerdo; AP = artéria pulmonar; \\
Cap = capilar pulmonar; AVP = átrio venoso pulmonar; $\varnothing$ túnel VP = diâmetro do túnel das veias pulmonares; VD = ventrículo \\
direito; Ao = aorta.
\end{tabular}

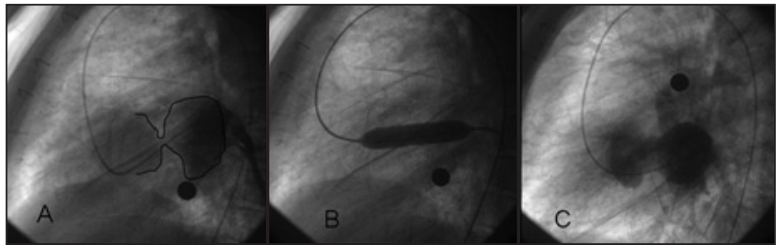

Figura 3 - Angioplastia com cateter-balão do túnel das veias pulmonares. A: Angiografia realizada através de via retrógrada, demonstrando estenose grave no terço médio da tunelização das veias pulmonares (área delineada). B: Insuflação do balão utilizado para a pré-dilatação. C: Resultado final, com nítida melhora da área estenótica.

ra significativa do gradiente intratúnel (Tabela 3 e Figuras 3B e 3C). Logo após a dilatação, a paciente apresentou grave edema agudo de pulmão, secundário à obstrução total da drenagem pulmonar durante a insuflação dos balões, tendo que ser ventilada com parâmetros ventilatórios altos para controle do quadro, que durou aproximadamente 15 minutos. Novas angiografias demonstraram melhora nítida do local da estenose (Tabela 3). Após o procedimento, a criança foi transferida para a Unidade de Tratamento Intensivo, onde foi extubada oito horas após o procedimento, sem dificuldades. A paciente recebeu alta hospitalar quatro dias após o procedimento, em uso de diuréticos. O ecocardiograma realizado antes da alta evidenciou gradiente na tunelização das veias pulmonares de $4 \mathrm{mmHg}$, boa função ventricular e valvas atrioventriculares competentes.

\section{DISCUSSÃO}

Durante as décadas de 1970 e 1980, a cirurgia de "correção fisiológica" no plano atrial utilizando as técnicas de Senning ou Mustard mudou a evolução natural da TGA, com bons resultados a curto e longo prazos. Inúmeras complicações, porém, foram descritas durante o seguimento, incluindo transtornos do ritmo cardíaco, disfunção ventricular direita e morte súbita.
As oclusões dos túneis intra-atriais, sistêmico ou pulmonar, são complicações potencialmente graves, sendo responsáveis por $30,7 \%$ das reoperações, como publicado por Lange et al. ${ }^{6}$, recentemente. O mecanismo principal da obstrução decorre da retração cicatricial das linhas de sutura. A oclusão isolada do túnel da VCS é a mais freqüente $(5 \% \text { a } 10 \%)^{7}$. Inicialmente, a maioria dos pacientes é assintomática, em decorrência de "descompressão" do sistema venoso através da veia ázigo. Os sintomas só aparecem na ausência desse sistema ou na estenose da VCI. Por outro lado, a obstrução do túnel da $\mathrm{VCl}$ é uma complicação rara $(<1 \%)$, porém potencialmente grave, porque pode afetar a circulação e a função hepática e levar à trombose da veia cava ou das veias que nela drenam. A estenose do túnel das veias pulmonares acontece em aproximadamente $2 \%$ dos casos e sua principal conseqüência é a hipertensão venocapilar acentuada, levando à deterioração respiratória progressiva dos pacientes ${ }^{7}$. As reoperações das estenoses dos túneis estão associadas a morbidade e mortalidade significativas. Segundo Wells e Blackstone ${ }^{5}$, a mortalidade total foi de $36 \%$ em uma série de 19 reoperações (12 obstruções de túneis, cinco leaks e duas disfunções do ventrículo direito). O tratamento percutâneo vem sendo utilizado na última década, com resultados positivos ${ }^{8-10}$.

Neste artigo, relatamos o tratamento percutâneo de dois pacientes com estenose grave dos túneis intraatriais. No primeiro caso, a estenose encontrava-se na tunelização de ambas as veias cavas, fato extremamente raro e grave. A decisão pela abordagem estagiada foi baseada principalmente no quadro clínico e na impossibilidade de abordar a VCl através das veias femorais. Seguindo a recomendação de alguns autores, foi realizado implante direto do stent no túnel superior, sem dilatação prévia9 ${ }^{9}$. O implante do stent no túnel da $\mathrm{VCl}$ através da punção trans-hepática é um procedimento inédito não relatado na literatura até 
o momento e sua indicação encontra-se justificada pela hipertensão venosa grave na parte inferior do corpo, que provavelmente levou à formação de trombo com oclusão total da $\mathrm{VCl}$, antes da origem das veias renais. Houve trombose inclusive da própria veia renal direita e, com isso, a perda funcional do rim, corroborado na fluoroscopia, caracterizada por ausência de contraste no sistema pielocalicial direito.

No segundo caso, a estenose do túnel das veias pulmonares representa também uma complicação rara e grave. Como pode ser observado na Tabela 3, a criança encontrava-se com hipertensão pulmonar secundária a estase venocapilar grave, sendo essa a indicação da intervenção. Nesse caso, é importante salientar que as angiografias obtidas através das artérias pulmonares não proporcionaram imagens satisfatórias, sendo necessária a abordagem "retrógrada", por via arterial, através da valva tricúspide. Optou-se pela angioplastia isolada, sem implante de stent, por causa da via de abordagem arterial, já que para o uso de stent é necessária a utilização de bainhas maiores, o que poderia ocasionar complicações arteriais numa criança considerada pequena $(35 \mathrm{~kg}$ ). Além disso, uma bainha longa teria que ser posicionada no átrio venoso pulmonar em forma "retrógrada", o que seria tecnicamente muito difícil. Mesmo utilizando-se essa via retrógrada não convencional para dilatação do túnel venoso pulmonar, houve sustentação adequada para a navegação dos balões. Além disso, é importante notar que não houve danos à valva tricúspide, o que teria sido potencialmente catastrófico nesse caso. O edema agudo de pulmão logo após dilatação reflete a pobre situação hemodinâmica e a gravidade em que a paciente se encontrava. Essa complicação, ao nosso entender, apesar de não ter sido relatada até o presente momento na literatura, é uma alteração "esperada" e transitória se a dilatação for completada com sucesso.

\section{CONCLUSÃO}

Apesar de as técnicas de correção atrial para TGA atualmente terem sido praticamente abandonadas, existe um grupo considerável de pacientes submetidos a esse tipo de correção em épocas anteriores e suas complicações tardias ainda estão presentes em nossa prática clínica atual. A obstrução dos túneis intraatriais é um achado relativamente freqüente e o tratamento percutâneo dos mesmos, mediante angioplastia com balão ou implante de stents, representa uma opção segura e eficaz em casos selecionados, como foi demonstrado neste artigo.

\section{REFERÊNCIAS BIBLIOGRÁFICAS}

1. DeLeon VH, Hougen TJ, Norwood WI, Lang P, Marx GR, Castaneda A, et al. Results of the Senning operation for transposition of the great arteries with intact ventricular septum in neonates. Circulation. 1984;70(3 Pt 2):I21-5.

2. Williams WG, Trusler GA, Kirklin JW, Blackstone EH, Coles JG, Izukawa T, et al. Early and late results of a protocol for simple transposition leading to an atrial switch (Mustard) repair. J Thorac Cardiovasc Surg. 1988;95(4):717-26.

3. Jatene AD, Fontes VF, Paulista PP, Souza LC, Neger F, Galantier $\mathrm{M}$, et al. Anatomic correction of transposition of the great vessels. J Thorac Cardiovasc Surg. 1976;72(3):364-70.

4. Hörer J, Haas F, Cleuziou J, Schreiber C, Kostolny M, Vogt $M$, et al. Intermediate-term results of the Senning or Mustard procedures combined with the Rastelli operation for patients with discordant atrioventricular connections associated with discordant ventriculoarterial connections or double outlet right ventricle. Cardiol Young. 2007;17(2):158-65.

5. Wells WJ, Blackstone E. Intermediate outcome after Mustard and Senning procedures: a study by the Congenital Heart Surgeons Society. Semin Thorac Cardiovasc Surg Pediatr Card Surg Annu. 2000;3:186-97.

6. Lange R, Hörer J, Kostolny M, Cleuziou J, Vogt M, Busch R, et al. Presence of a ventricular septal defect and the Mustard operation are risk factors for late mortality after the atrial switch operation: thirty years of follow-up in 417 patients at a single center. Circulation. 2006;114(18):1905-13.

7. Williams WG, Trusler GA, Kirklin JW, Blackstone EH, Coles JG, Izukawa T, et al. Early and late results of a protocol for simple transposition leading to an atrial switch (Mustard) repair. J Thorac Cardiovasc Surg. 1988;95(4):717-26.

8. Kajita LJ, Brito Júnior FS, Veloso WU, Rati MA, Arie S. Balloon dilatation of stenosed pulmonary venous atrium after the Senning procedure. Am Heart J. 1995;129(3):618-20.

9. Tzifa A, Marshall AC, McElhinney DB, Lock JE, Geggel RL. Endovascular treatment for superior vena cava occlusion or obstruction in a pediatric and young adult population: a 22-year experience. J Am Coll Cardiol. 2007;49(9):1003-9.

10. Daehnert I, Hennig B, Wiener M, Rotzsch C. Interventions in leaks and obstructions of the interatrial baffle late after Mustard and Senning correction for transposition of the great arteries. Catheter Cardiovasc Interv. 2005;66(3):400-7. 